\title{
TOPOLOGICAL DYNAMICS IN COSET TRANSFORMATION GROUPS
}

\author{
BY HARVEY B. KEYNES ${ }^{1}$
}

Communicated by G. A. Hedlund, July 6, 1966

1. Introduction. In [1], the actions of the 1-parameter subgroups on coset spaces of certain types of Lie groups were studied with regard to various properties occurring in topological dynamics. A central question was to determine when these actions were minimal and distal. By a considerable use of various structure theorems of Lie groups, it was shown that if the Lie group is connected simplyconnected and nilpotent, and the subgroup is discrete and syndetic, then the action of any 1-parameter subgroup is always distal [1, IV, Theorem 3]. Moreover [1, IV, Theorem 5], the action is minimal for those 1-parameter subgroups induced by elements of a comeager subset of the Lie group. In this paper, we use only the properties of topological groups and various results in topological dynamics to generalize the first result. Moreover, we show that the second result can be formulated more generally in the setting of a topological group, and we recover the result in an alternate way.

Let $(X, T)$ be a transformation group. A subset $A$ of $T$ is (left) syndetic if there is a compact subset $K$ of $T$ such that $T=A K$. A point $x$ of $X$ is transitive if $\operatorname{cl}(x T)=X$. If some point of $X$ is transitive, then $(X, T)$ is a point-transitive transformation group. If every point of $X$ is transitive, then $(X, T)$ is a minimal transformation group. If $X$ is a uniform space and $x, y \in X$, then $x$ and $y$ are proximal provided that for every index $\alpha$ of $X$ there exists $t \in T$ such that $(x t, y t) \in \alpha$. The transformation group $(X, T)$ is distal if every pair of distinct points of $X$ is not proximal. As a general reference for these notions, see [2].

Let $G$ be a topological group and let $H$ be a subgroup of $G$. Then $H \backslash G$ will denote the space of right cosets of $H$ in $G$. The coset transformation group of $G$ induced by $H$ is the transformation group $(H \backslash G, G)$ with action $(H f, g) \rightarrow H f g$.

Throughout this paper, $G$ will denote a topological group, and $H$ will denote a (left) syndetic closed subgroup of $G$. We will utilize right-handed functional notation.

${ }^{1}$ These results form a part of a doctoral dissertation written at Wesleyan University under the supervision of Professor W. H. Gottschalk. During this period, the author held a National Science Foundation Cooperative Graduate Fellowship. 
2. A general criterion for distal. A crucial remark to the proof of the main result is the following:

(2.1) REMARK. Let $(X, T)$ and $(Y, T)$ be transformation groups with compact uniform phase spaces $X$ and $Y$. Let $f$ be a transformation group homomorphism from $(X, T)$ onto $(Y, T)$. For all $y \in Y$, let $P(y)$ be the period of $Y$ at $y$. Suppose that $(Y, T)$ is a distal and pointwise periodic transformation group. Suppose further that for all $y \in Y,\left(y f^{-1}\right.$, $P(y))$ is a distal transformation group. Then $(X, T)$ is a distal transformation group.

(2.1) roughly states that we can enlarge the phase space and still maintain a distal action.

Let $K$ be a subgroup of $G$. Then $K$ is subnormal in $G$, denoted by $K \triangleleft \triangleleft G$, if there exists a finite sequence $\left(K_{0}, K_{1}, \cdots, K_{n}\right)$ of subgroups of $G$ such that $K=K_{0} \triangleleft K_{1} \triangleleft \cdots \triangleleft K_{n}=G$.

We now state the main result of this section.

(2.2) Theorem. Let $H \triangleleft \triangleleft G$. Then $(H \backslash G, G)$ is a distal transformation group.

Since it is well known (see [4, Theorem (6.4.10)]) that every subgroup of a nilpotent group is subnormal, we immediately obtain:

(2.3) Corollary. Let $G$ be a nilpotent group. Then $(H \backslash G, G)$ is a distal transformation group.

It is easy to see that if $(X, T)$ is a distal transformation group, then every subgroup of $T$ also yields a distal action on $X$. Thus, [1, IV, Theorem 3] is an immediate corollary of (2.3).

3. The existence of point-transitive, and minimal distal subgroups. Let $T$ be a topological group. Let $K$ be a subgroup of $G$. Let $\theta$ be a topological group homomorphism of $T$ into $G$. Then the coset transformation group induced by $\theta$ is the transformation group $(K \backslash G, T, \pi(\theta))$ with action $(K g, t) \pi(\theta)=K g(t \theta)$. Let $\operatorname{Hom}(T, G)=[\theta \mid \theta: T \rightarrow G$ is a topological group homomorphism from $T$ into $G$ ], provided with its point-open topology. Then $\operatorname{Hom}(T, G)$ [covers] [continuously covers] $G$ if there exists a [map] [continuous map] $\theta: G \rightarrow \operatorname{Hom}(T, G)$, where $g \rightarrow \theta_{g}$, such that for all $g \in G, g \in T \theta_{g}$. Such a map $\theta$ is called a [covering] [continuous covering] choice funciion.

Using a Baire category argument, we can show:

(3.1) Theorem. Let $G$ be a locally compact second countable group, let $K$ be a subgroup of $G$, let $T$ be a topological group, let $\theta: G \rightarrow \operatorname{Hom}(T, G)$, where $g \rightarrow \theta_{g}$, be a continuous covering choice function, and let $f \in G$. Sup- 
pose that for every nonvacuous open subset $U$ of $G, G=\operatorname{cl}\left(K f\left(\bigcup_{g \in U} T \theta_{g}\right)\right)$. Then for almost all $g \in G$ (i.e., for $g$ in a comeager subset of $G$ ), $K f$ is a transitive point of $\left(K \backslash G, T, \pi\left(\theta_{g}\right)\right)$.

Let $\boldsymbol{Z}$ be the additive group of all integers. Let $g \in G$. Then $\boldsymbol{\phi}_{g}: \boldsymbol{Z} \rightarrow G$ is the topological group homomorphism defined by $n \phi_{g}=g^{n}$. If $A \subset G$, then $A^{*}$ will denote the set $\bigcup_{g \in A} \boldsymbol{Z} \phi_{g}$.

By using the cannonical continuous covering choice function $\phi: G \rightarrow \operatorname{Hom}(\boldsymbol{Z}, G)$, where $g \rightarrow \phi_{g}$ and applying (2.2), (3.1) yields:

(3.2) Theorem. Let $G$ be a locally compact second countable group, let $T$ be a topological group, and let $\theta: G \rightarrow \operatorname{Hom}(T, G)$, where $g \rightarrow \theta_{g}$, be a covering choice function. Suppose that $H \triangleleft \triangleleft G$. Suppose further that there exists $f \in G$ such that for every nonvacuous open subset $U$ of $G$, $G=\operatorname{cl}\left(H f U^{*}\right)$. Then:

1. For almost all $\mathrm{g} \in G,\left(H \backslash G, \boldsymbol{Z}, \pi\left(\phi_{\theta}\right)\right)$ is a minimal distal transformation group.

2. For almost all $g \in G,\left(H \backslash G, T, \pi\left(\theta_{g}\right)\right)$ is a minimal distal transformation group.

Now let $G$ be a connected simply-connected nilpotent Lie group, and let $H$ be discrete. It is known [3] that the exponential map is a homeomorphism. If $R$ denotes the additive group of all real numbers, let $\theta: G \rightarrow \operatorname{Hom}(R, G)$ be the covering choice function induced by the 1-parameter subgroups. It follows by [1, Lemma, pp. 55-56] that for every nonvacuous open subset $U$ of $G, G=H U^{*}$. Then $[1, \mathrm{IV}$, Theorem 5 and Corollary] is a consequence of (2.3) and (3.2).

Complete proofs of these results will be given in a future paper.

\section{REFERENCES}

1. L. Auslander, L. Green, F. Hahn, et al., Flows on homogeneous spaces, Annals of Mathematics Studies No. 53, Princeton Univ. Press, Princeton, N. J., 1963.

2. W. Gottschalk and G. Hedlund, Topological dynamics, Amer. Math. Soc. Colloq. Publ., Vol. 36, Amer. Math. Soc., Providence, R. I., 1955.

3. A. Malcev, On a class of homogeneous spaces, Amer. Math. Soc. Transl. (1) 39 (1951), 33 pp.

4. W. R. Scott, Group theory, Prentice-Hall, Englewood Cliffs, N. J., 1964.

WESLEYAN UNIVERSITY and

University of California, Santa Barbara 